\title{
Quality and Availability of Spectrum based Routing for Cognitive Radio enabled IoT Networks
}

\author{
M.K. Kaushik, Y. Yoganandam and SK Sahoo
}

\begin{abstract}
With the recent emergence and its wide spread applicability Internet of Things (IoT) is placing pressure on network resources and most importantly on availability of spectrum. Spectrum scarcity is the issue to be addressed in networking within IoT. Cognitive radio is the technology which addresses the problem of spectrum scarcity in an efficient way. Equipping the IoT devices with cognitive radio capability will lead to a new dimension called cognitive radio enabled IoT devices. To achieve $\mathrm{ON}$-demand IoT solutions and interference free communications cognitive radio enabled IoT devices will become an effective platform for many applications. As there is high dynamicity in availability of spectrum it is challenging for designing an efficient routing protocol for secondary users in cognitive device networks. In this work we are going to estimate spectrum quality and spectrum availability based on two parameters called global information about spectrum usage and instant spectrum status information. Enhanced energy detector is used at each and every node for better probability of detection. For estimating spectrum quality and availability we are introducing novel routing metrics. To have restriction on the number of rerouting and to increase the performance of routing in our proposed routing metric only one retransmission is allowed. Then, two algorithms for routing are designed for evaluating the performance of routing and we find that the bit error rates of proposed algorithms (nodes are dynamic) have decreased a lot when compared to conventional methods (nodes are static) and throughput of proposed algorithm also improved a lot.
\end{abstract}

Index Terms - cognitive radio networks; Internet of Things; Spectrum availability; Spectrum sensing.

\section{INTRODUCTION}

In the existing wireless and cellular networks only few devices are connected viz., tablets, smart phones and laptops. But Internet of things (IoT) includes many variety of sensors like

Manuscript received May 15, 2018; revised August 24, 2018. Date of publication September 17, 2018. Prof. Nikola Rožić has been coordinating the review of this manuscript and approved it for publication.

M.K. Kaushik and Y. Yoganandam are with the Department of Electrical Engineering, BITS Pilani Hyderabad Campus, Hyderabad, India. (e-mails: \{kaushiknicky24, yoganandamy\}@gmail.com). SK Sahoo is with the SPM International Institute of Information Technology, Naya Raipur, India. (e-mail: sahooemailid@gmail.com).

Digital Object Identifier (DOI): 10.24138/jcomss.v14i3.557 health monitoring devices, autonomous cars, home appliances, environmental sensors, smart meters and many [1], [2]. Concept of IoT enabled human to machine and machine to machine communications are discussed in [3].

There are so many application areas in IoT paradigm which includes smart manufacturing and precision agriculture. In order to develop the IoT solutions lot of work is to be done to design networking components. As the sensors in IoT possess different software, hardware and network types, refinement of networking components is required in order to work together.

As deployment of devices and mobile applications are increasing day by day the unlicensed Industrial scientific and Medicine (ISM) bands are crowded heavily. The Federal communications commission has mentioned that most of the spectrum is static allocated in certain geographical regions and hence large portions of the spectrum are underutilized [4]. To have an efficient usage of spectrum cognitive radio has been introduced [5]-[8]. In cognitive radio networks (CRNs) primary users and secondary users coexist to transfer their data. Secondary users are usually cognitive devices which will sense the surroundings and make an opportunistic decision on which band they have to transfer their data packets. In doing so secondary users must not interfere with primary users. Whenever there is a hint of primary user coming back, secondary user has to switch the spectrum band to continue its data transfer.

Nowadays multi-hop cognitive device networks (CDNs) are gaining popularity [9]-[14]. In multi hop CDN, multiple IoT devices are present which are equipped with cognitive radio technology and communicate among themselves over the available spectrum bands which are opportunistically chosen from primary users in a multi hop manner [15]. In multi hop CDN the cognitive devices will perform spectrum sensing method and choose the appropriate channels which are declared idle by spectrum sensing methods and choose another channel immediately when the sensing algorithm identifies that primary user wants to use the channel. With the help of cognitive radio technology multi hop CDNs or cognitive enabled IoT devices will increase spectrum efficiency, utilization of spectrum and network lifetime [15], [16]. 
Amongst different research surveys in networking, routing plays an important role and hence it has got attention in research groups. In order to transfer data packets from source to destination routing plays an important role. Much of research has happened on routing techniques [17]-[20] for wireless sensor networks and existing CDNs. If these routing techniques are directly implemented in multi hop CDNs or cognitive enabled IoT devices these routing techniques will fail. Because of mobility of devices in the network, routing in multi hop CDNs become a challenging task [21] - [23]. First, in multi hop CDNs there won't be any common channel which could be used by all secondary users (SU) between source SU and destination SU. In the traditional and existing routing schemes the goal was to identify path which can be accessed by all the secondary users with one common available channel. On the other side there exists a path where neighboring secondary users will share a common available channel even though global common channel does not exist along the path. Second, as the availability of spectrum changes randomly for secondary users it is preferable to estimate spectrum availability from local and global views. We know that rerouting is required whenever primary user comes back while secondary user is transmitting its data packets. Hence in multi hop CDNs routing performance will greatly decrease due to rerouting and spectrum hand offs. Therefore in our work it is important to select a routing path which has less number of rerouting which requires consideration of temporarily unavailable spectrum. Lastly for optimal selection of routing path, maintenance of spectrum quality (spectrum average idle time, spectrum bandwidths) is required. A selected path may have multiple bands with their own spectrum availability and quality. When the data transmission is happening the band which is chosen will become invalid with the arrival of primary users. Instantly new band has to be chosen which might be available due to primary users exit. Hence it is required to assess the quality of available and unavailable spectrum for a good routing scheme. The above mentioned scenarios has to be kept in mind to design a routing scheme in Cognitive radio enabled IoT devices.

The contributions made in this paper are summarized as follows 1) Enhanced energy detector is used at each and every node for better probability of detection. 2) For estimating spectrum quality and availability we are introducing novel routing metrics in section $\mathrm{V}$. 3) To have restriction on the number of re-routings and to increase the performance of routing in our proposed routing metric only one retransmission is allowed. 4) Two algorithms for routing are designed for evaluating the performance of routing and we find that the bit error rates of proposed algorithms (nodes are dynamic) have decreased a lot when compared to conventional methods (Nodes are static) and throughput of proposed algorithm also improved a lot

The rest of this paper is organized as follows: Section II discusses about motivation towards developing Cognitive radio based IoT networks. Some applications of CR based IoT and the requirement of standardization is discussed in Section III. Section IV discusses about the related works in the literature. Section V and VI discusses about System model and problem formulation respectively. Proposed routing algorithm is explained in Section VII. The simulation results are presented in Section VIII and our findings are summarized in Section IX.

\section{Motivation}

Underutilization of spectrum is clearly evident [24] in existing fixed utilization of spectrum policies. To avoid underutilization of spectrum cognitive radio networks (CRN) play an important role and hence they have become popular these days. Cognitive radio networks work in an opportunistic manner where it interacts with the surrounding environment by modifying parameters of transmitter based on the interactions. Every node in the CRN has ability to switch fast with instantaneous transmissions in addition to learning channel state information and channel probing. As the spectrum is accessed dynamically cognitive user can adapt to changing network conditions. Cognitive radio has to use the spectrum on a condition that it will not interfere licensed or primary user.

With the advancements in the development of IoT and CRNs it is intended that cognitive radio based IoT structures will become crucial requirements in the future. By understanding physical and social worlds [24], [25], [26] IoT has to be equipped with intelligence or cognition to learn, think and to make decisions. It also has to make cognitive decisions, perception analysis, substantial data analytics and providing service on demand. Hence cognitive radio based IoT is required in the coming future, might be due to the reasons given below: 1) Spectrum allocation for IoT devices is the prime motivation for cognitive radio based IoT. IoT devices are going to increase in great numbers, it becomes a tedious job to allocate spectrum bands to these devices. It is known fact that primary users are increasing and their requirements also, which creates problems for cognitive users. Therefore assigning spectral bands for large number of devices with existing fixed spectrum allocations can lead to excessive expenditures. As conventional communication methods will not support sharing of spectrum between multiple users CRNs can be used in these scenarios. CRNs has advantage of spectrum sharing among multiple IoT devices [26], [27]. With cognitive capabilities CRNs will make intelligent decisions among multiple devices.

2) A CRN standard, IEEE 802.22, has maximum range and it is suitable for applications which are in short to long range applications [26], [27].

3) The capability of accessing spectrum dynamically will lead cognitive radio based IoT networks be in interference free channels.

4) In IoT structures/Networks many sensors are equipped on objects which are movable, hence it will become a difficult task to maintain continuous communication facility all the time. To have seamless connectivity [28], [29] IoT devices are equipped with cognitive capabilities.

5) As IoT devices generate huge data a new standard of cloud servers are gaining huge popularity. Therefore if IoT has cognitive capabilities it will search for storage spaces in cloud servers by itself and with the help of spectrum sensing it will send the data [28], [29].

\section{StANDARDIZATION AND APPLICATIONS OF CR BASED IOT}

To get IoT into commercial and personal spaces standardization is required. There are so many working groups, standardization bodies and technical committees are working together to bring IoT into realization viz., Internet engineering task force, IEEE, National institute of standards technology, 
European Telecommunications standards institute and Third generation partnership project. So much of efforts are put into developing a communication protocols for IoT. It is known fact that cognitive radio will support multiple users without interference in spectrum underutilized space. All the applications which comes under personal, commercial and industrial applications will get benefitted from CRNs. IEEE 802.22 is the first international standard which operates in television white space. To realize cognitive radio based IoT, immense efforts are required. As there are many new bands opening up for CRNs placing IoT in this structure will be a great choice. Another option would be coexistence of cognitive radio based IoT in current regulatory bodies. Research happening in IoT standards are in short range techniques such as radio frequency identification (RFID) and near field communication (NFC) but CRN standardization has happened in short range to medium range as well as long range standards like television white spaces. Combination of both will serve so many future applications with high efficiency and within limited spectrum. Few applications of IoT which can get benefitted from CRNs are described below:

\section{A. Healthcare}

IoT based healthcare applications are at present practically there in the market. In order to observe critical data such as glucose level, temperature, blood pressure and others of a patient, sensors are deployed. Medical workers will continuously monitor the parameters and provide solutions wirelessly. For this purpose spectrum assignment wouldn't be an issue. Cognitive radio based IoT networks would help in this regard without concerning about availability of spectrum [30].

\section{B. Social Activities}

Applications like electronic tolling and traffic monitoring has drawn much attention in intelligent transport systems which includes sensors based on road and vehicle. For long coverage applications not much of research has happened. CRNs will solve these problems in a more suitable way [28].

\section{Applications related to Environment}

Monitoring of $\mathrm{CO}_{2}$ emissions, waste management, estimations on humidity, temperature and noise measurements are some of the applications which are related to environmental domain. Huge number of heterogeneous devices must be installed at important junctions for efficient and reliable monitoring. In these scenarios spectrum assignment would become a problem with the current static assignment policy. With the addition of cognitive capabilities to the IoT functionalities a great solution would come for congested areas [28].

\section{Home Applications}

Smart fridge and smart lights are examples for home automation and home energy management. Many sensors are installed to perform every day functions for enhanced quality of life. Generally WiFi access points are used for these applications; but these will cause heavy interference in the industrial, scientific and medical bands. A reliable solution would be to make sensors with cognitive capabilities to avoid interference [28].

\section{E. Smart cities}

For enhanced life style smart city is the solution in an ecofriendly manner. For this continuous connectivity is must. User interaction upon data gathering is also an important factor. CRNs will help in the issue of continuous connectivity [31].

\section{F. Internet of vehicles:}

The objective of this is controlling a vehicle through the integration of embedded systems, communications and controls. Autonomous decision making for travelling is expected in internet of vehicles. By exchanging information from vehicles to vehicles, sensors on vehicles will make navigation safe in the future. The main challenge in Internet of vehicles is spectrum availability for mobile vehicles, CRNs will be an efficient solution for interference free spectrum sensing and long range communications[29], [32].

\section{G. Smart grid}

Consumers want to know about their energy consumption at any time and any place which indicates that IoT based smart grid is required in the future. One disadvantage in this is transferring large amounts of data from number of meters without interference to long distances that too in limited bandwidth. There are issues with existing wireless techniques. Wired techniques like optical fiber and digital subscriber line (DSL) can address the problem, but require huge expenditures for fiber or cable installation. Hence CRNs is a good solution [28].

\section{RELATED WORKS}

With the introduction of accessing the spectrum dynamically researchers have started utilizing cognitive radio technology in CDNs/Cognitive radio based IoT. Good amount of research can be found in [15], [33]. By applying different metrics in routing several approaches are proposed [8], [23], [33]-[42]. Therefore routing on demand opportunistically comes under two categories:

\section{A. Routing on demand in CDNs}

From a global point of view selection of a path from multiple paths is a prime focus in routing on demand CDNs (Adhoc On Demand Distance Vector (AODV) [43]). In AODV, path can be discovered on demand by licensed user. In [23] a routing protocol is designed which is aware of spectrum availability (SEARCH-spectrum aware routing protocol for cognitive adhoc networks) where broadcasting of route requests on each channel is done using Greedy Geographic strategy, then the secondary user at the destination choose a path which has least number of hops to the source and minimum interference with primary users. As SEARCH algorithm doesn't have ability to estimate availability of spectrum, route selection is correlated with changes in application. In [44] a shortest path algorithm with spectrum hand off scheduler and rerouting technology is proposed which can be applied on the primary users arrival. In [33] authors proposed an estimation technique of the spectrums available between any two secondary users which leads to success probability maximization in multi hop networks. It is known fact that the availability of spectrum is dynamic, due to this the rerouting will increase in [33] which causes degradation of route performance. Geographic routing protocol is proposed 
in [34] where secondary user will choose an optimal path which is shortest in distance to destination. This algorithm will perform well in sparse applications where there is less activity of primary users but it will behave poorly in applications where the availability of spectrum is dynamic.

\section{B. Opportunistic Routing in CDNs}

The prime idea behind routing opportunistically in CDNs is to find out the neighbors who has priority orders for every intermediate node. At the network layer each secondary user broadcasts data packets to the neighbors, whereas only one secondary user will respond and act as next relay in MAC layer. In [35] a routing protocol is proposed in which relay node priority is calculated by its spectrum position and quality such as channel reliability, channel throughput and distance to destination. A cross layer distributed opportunistic routing protocol is proposed in [37] where the selection of relay and sensing of spectrum are considered jointly for decreasing the delay in delivery rate. In [45] a routing protocol is proposed which is aware of spectrum and energy efficient to analyze the problem of optimizing spectrum usage efficiency and dynamics of spectrum. With this technique energy consumption and delivery latency are decreased. Though retransmissions are decreased by using broadcasting mechanisms in opportunistic routing methods they fall sometimes in local optimization in CDNs because availability of spectrum is not considered from global and local point of view.

\section{SYSTEM MODEL}

\section{A. System Model}

In this section we first give network model of cognitive radio based IoT networks (CDNs) and then present routing metrics followed by the problem formulation. In the previous works [46] the network model considered was multi hop CDN consisting static primary users and secondary users. In our work we are considering multi hop CDN with dynamic primary users and secondary users. At each and every secondary user node enhanced energy detector [47] is adopted for greater probability of detection instead of conventional energy detector. In the proposed CDN model, transmission of data will happen in multi hop manner when the distance from source and destination is greater than the transmission range of the source. To have easier routing, we consider, for any pair of secondary users there is at least one route path is present.

In a CDN only one channel can be accessed by each primary user from an orthogonal channel set $\mathrm{C}=\{\mathrm{C} 1, \mathrm{C} 2, \ldots . \mathrm{Cn}\}$. A common control channel exists from which controlling messages of secondary users are exchanged. With the addition of common channel, every secondary user equipped with half duplex CR [48] can access and switch to any primary user channels which are available. In a $\mathrm{CDN}$, the secondary users find out the frequency channels that are not utilized by primary users with the help of spectrum sensing. Every secondary user makes a choice of possible control channel after sensing spectrum holes. The possible control channel is selected based on a quality metric which is interference-power constraint [50] imposed by primary users. The interference constraint is less with a higher channel quality such that the channel is available for more time for cognitive user transmission. Each secondary users prefer frequency channels with better quality as the control channel. As mentioned before secondary user has to vacate the channel and search for one more available channel whenever primary user comes back and claims for the channel. For easy routing on a given channel secondary users are assumed to transfer data with fixed transmission power. An ON-OFF model is proposed in the reference [49], where a renewal process is introduced to formulate the activities of primary users. In ON-OFF model channel accessing follows a poisson process, where ON period is represented as $\mathrm{T}^{\mathrm{k}}$ ON with $\lambda_{\mathrm{k}}$ rate and $\mathrm{T}^{\mathrm{k}}{ }_{\text {OFF }}$ is represented for OFF period with $\mu_{\mathrm{k}}$ rate. Hence $\mathrm{T}^{\mathrm{k}}$ ON and $\mathrm{T}^{\mathrm{k}}$ OFF are used to represent primary users availability and non-availability to access channel $\mathrm{k}$.

\section{B. Routing Metrics}

In this section routing metrics are discussed from statistical point of view only, where no current spectrum available information is not considered, and then we introduced proposed routing metrics by considering global statistical data and current spectrum availability (instant information).

\section{1) When instant information is not considered}

With the help of alternating renewal process method limiting probability can be obtained. Where at any given time a primary user i cannot access a channel $\mathrm{k}$. We represent it as $\mathrm{P}^{\mathrm{k}} \mathrm{OFF},{ }_{i}$ which can be given as

$$
P_{o f f, i}^{k}=\frac{\mu_{i, k}}{\mu_{i, k}+\lambda_{i, k}}
$$

Where $\lambda_{\mathrm{i}, \mathrm{k}}$ and $\mu_{\mathrm{i}, \mathrm{k}}$ denotes the rate of PU i using $\mathrm{k}$ and PU i not using $\mathrm{k}$ respectively. The meaning of limiting probability is inferring that a given PU i does not utilize channel $\mathrm{k}$ at any time with probability $\mathrm{P}^{\mathrm{k}}{ }_{\text {off }, \mathrm{i}}$ even if we do not obtain the actual spectrum usage information about PU i on channel k. similarly, $\mathrm{P}^{\mathrm{k}}{ }_{\text {on, } \mathrm{i}}$ is denoted to infer the probability a given PU being in active state over a given channel.

With the help of shannon capacity theorem the transmission rate which is achievable through channel $\mathrm{k}$ from secondary user i to its neighboring secondary user is given as follows

$$
V_{k}=B+\log _{2}\left(1+\frac{P_{i, j}^{k}}{B N_{0}}\right)
$$

Where $\mathrm{P}_{\mathrm{i}, \mathrm{j}}^{\mathrm{k}}, \mathrm{N}_{0}, \mathrm{~V}_{\mathrm{k}}$ and $\mathrm{B}$ represent power received by secondary user $\mathrm{j}$, thermal noise, transmission rate and channel bandwidth respectively. Usually $\mathrm{P}_{\mathrm{i}, \mathrm{j}}$ is inversely proportional to distance between $\mathrm{SU}$ i and $\mathrm{SU} \mathrm{j}$ and directly proportional to transmission power of SU i. As it is assumed that each secondary user has fixed transmission power, the rate of transmission is sensitive to distance between two secondary users. SU will take minimum $S / V_{k}$ to transmit data over channel $\mathrm{k}$, where $\mathrm{S}$ is the size of data and $\mathrm{V}_{\mathrm{k}}$ represents achievable data transmission rate over channel $\mathrm{k}$.

As it is known fact that secondary users has to empty the channel upon the arrival of primary user, and hence total transmission delay will increase. Therefore successful transmission probability is dependent on both spectrum availability time and required transmission time. From [33] the delivery success probability of secondary user $i$ to its peer secondary user $\mathrm{j}$ can be given as 


$$
\begin{aligned}
P_{s u c}^{k}(i, j) & =P\left(T_{k} \geq s / v_{k}\right) \\
& =e^{-s /\left(v_{k}, \mu_{k}\right)}
\end{aligned}
$$

Where $\mathrm{V}_{\mathrm{k}}$ is the amount of transmission through channel $\mathrm{k}, \mu_{\mathrm{k}}$ is the rate of channel $\mathrm{k}$ which is unavailable and $\mathrm{S}$ is given as packet size.

It is clearly observed that the above metric considers statistical information about spectrum but not including the instant information about usage of spectrum, like if the channels are utilized by primary users or not. Existing routing metrics (e.g. [33]) will consider maximum probability of successful transmission but not considering the potential channels. As we all know that due to dynamicity of primary users arrival, retransmitting and rerouting will happen when the primary user comes back. It is known fact that retransmitting and rerouting will decrease the routing performance and increase the transmission delay. So in the proposed routing metric by considering above mentioned cases minimization of retransmitting and rerouting is done. Hence it will be better to consider spectrum usage which is calculated instantly and global information about spectrum in multi hop CDNs. Now we are going to propose our routing metrics based on the above discussed factors.

\section{2) Proposed Routing metrics}

In a multi hop CDN secondary user will select a channel for transmitting its data packets from the set of available channels. When there is any interruption in the last data transmission, secondary user can always retransmit data packets on other channel if transmission delay is not considered. Therefore with the increased number of retransmissions, data transmission will succeed finally. Even in the case of one channel availability between two secondary users, transmission can be completed by secondary user waiting till channels next availability. But this will fail in real time applications because of time-to-live (TTL) limitation for any packet will be abandoned when TTL is equal to zero. That is why it is important to consider retransmission in routing in a multi hop CDN.

If a condition is considered that number of transmission is 1 . On any two neighboring secondary users the transmission probability over all the available channels is given as 1-(1-p1) (1-p2)..... (1-pn). Where $\mathrm{n}$ stands for current available channels and $\mathrm{P}_{\mathrm{k}}=\mathrm{P}_{\text {suc }}^{\mathrm{k}}(\mathrm{i}, \mathrm{j})$ is represented as delivery success probability from channel $\mathrm{k}$. It is fact that with the increase in number of available channels delivery success probability also will increase. But the formula mentioned above is not accurate because the channel availability will change dynamically when transmission of data is happening.

As retransmission is restricted to 1 the delivery success probability from secondary user i to neighboring secondary user $\mathrm{j}$ on all channels is given as

$$
P_{\text {suc }}(i, j)=P_{0}+\left(1-P_{0}\right) \cdot \sum_{k=1}^{n-1}\left(V_{k} \cdot \prod_{m=1}^{k-1}\left(1-V_{m}\right) P_{k}\right)
$$

Where $\mathrm{n}$ indicates channels among secondary users $\mathrm{i}$ and $\mathrm{j}$, $\mathrm{P}_{0}=\mathrm{P}^{\mathrm{k}}$ suc $(\mathrm{i}, \mathrm{j})$ is the major success probability and $\mathrm{V}_{\mathrm{k}}$ represents probability of remaining channel $\mathrm{k}$ available if the first transmission is failed. In our work secondary user will access channel which has major delivery success probability to enhance the probability of transmission.
If secondary user fails at the first time for transmitting data on channel $\mathrm{k}$, channel availability will come under two cases: unavailable and available. By keeping in mind about the two states of $\mathrm{k}$ channel $\mathrm{V}_{\mathrm{k}}$ is given as

$$
V_{k}=\max \left(e^{-s /\left(2 v_{k} \mu_{k}\right)}, \frac{\mu_{k}}{\mu_{k}+\lambda_{k}}\right)
$$

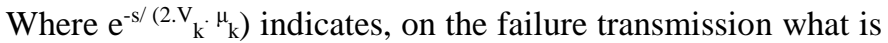
the probability that channel $\mathrm{k}$ still remains accessible, and $\mu_{\mathrm{k}} / \mu_{\mathrm{k}}+\lambda_{\mathrm{k}}$ indicates probability of channel $\mathrm{k}$ remains accessible.

With the constraint of retransmission initial routing metric is presented which represents delivery success probability for a path $\mathrm{P}$.

$$
T S P_{p}=\min _{(i, j) \in P} P_{s u c}(i, j)
$$

Where (i, j) represents secondary user $\mathrm{i}$ and its neighbor secondary user $\mathrm{j}$ in path $\mathrm{P}$.

If the packet is transferred in two attempts successfully then transmission delay from secondary user $i$ to secondary user $j$ using all the channels, $T_{(i, j)}$ is given as

$$
T_{i, j}=\frac{S}{V_{0}} \cdot P_{0}+\left(1-P_{0}\right) \cdot \sum_{k=1}^{n-1}\left(\left(\frac{S}{2 V_{0}}+\frac{S}{V_{k}}\right) \cdot V_{k} \cdot \prod_{m=1}^{k-1}\left(1-V_{m}\right) \cdot P_{k}\right)
$$

Where $s / v_{0}$ is given as average time over a channel with high transmission probability. Therefore value $s / 2 v_{0}$ is average time when the first transmission fails. Second, new routing metric in a path which estimates total average transmission delay in a path can also be obtained.

Transmission time delay over path $\mathrm{P}$ when a packet is transferred in two attempts, $\mathrm{TTD}_{\mathrm{P}}$ is given as

$$
T T D_{p}=\sum_{(i, j) \in p} T_{i, j}
$$

Where $\mathrm{TTD}_{\mathrm{P}}$ is the summation of transmission delays over all neighboring secondary users in the path $\mathrm{P}$. Therefore both instant availability of spectrum and statistical availability of spectrum have been considered in the above routing metrics.

\section{PROBLEM Formulation}

As per the discussions about two routing metrics, routing problem in CDNs can be viewed as the goal to optimize the routing metrics [46].

1) Under the condition that only one retransmission is allowed with delivery success probability metric can be resolved as follows:

$$
\max _{p} T S P_{p}
$$

2) Under the condition that only one transmission allowed with transmission delay metric can be resolved as follows:

$$
\min _{p} T T D_{p}
$$

\section{PROPOSED Routing Algorithm}

By considering both instant availability of spectrum information and statistical information about usage of spectrum, two new algorithms are proposed by including enhanced energy detector at each and every node and mobility of secondary users when compared with [46]. One is success transmission probability (maximization) and average delay in transmission (minimization) based on the routing metrics discussed above. 


\section{A. Success transmission probability (Maximization)}

The details of success transmission probability (Maximization) is illustrated in Algorithm 1. In this routing algorithm the aim is to find the best route with respect to maximizing the delivery success by considering only retransmission. The source secondary user will broadcast the route request message (RREQ) through common control channel in AODV way to destination secondary user. The success probability of each secondary user can be obtained by (6) is added along the path in RREQ message. Destination will select one route from number of RREQ messages by calculating the optimal path which has good success probability in delivering the data packets, along the path which is been chosen with the help of route reply message.

It is observed that the first method determines only the best path over which success probability is maximum in delivering data packets and will not determine actual channels in that particular path. As the spectrum resources are dynamically available each relay node can choose the channel with maximum success probability in delivering data packets from the channels which are all available. If there is a case that there are no available channels rerouting is done by relay secondary user.

$\underline{\text { Algorithm } 1 \text { Success transmission probability (Maximization) }}$ Input: parameters in multi-hop CSN, destination SU D, source SU S

Output: the route path which has largest success transmission probability

1: Source SU S creates RREQ messages $m$

2: The messages $m$ are broadcasted from Source SU S through

CCC channel to all neighboring SUs

3: for all each $\mathrm{SU} n$ which receives $m$ do

4: if SU $n$ is the destination SU D then

5: SU n obtains TSPp by Equation (6)

6: else

7: SU n obtains Psuc(I, j) by Equation (3)

8: SU $n$ forwards $m$ on over CCC channel

9: end if

10: end for

11: SU D waits for a predefined time upon receiving the first replica of message $m$

12: SU D determines the best route path which has the largest TSPp by using Equation (9)

13: SU D sends an acknowledgement back to $\mathrm{S}$ along the chosen path.

\section{B. Average delay in transmission (Minimization)}

The details of average delay in transmission (Minimization) is illustrated in Algorithm 2. In this routing algorithm the path is chosen by applying second routing metric. The selection of route is same as the above method where secondary user source will first broadcast the RREQ message through common control channel in an AODV way and forwarded to the destination. The additional inclusion in this method is during broadcasting information about spectrum regarding transmission delay by using (7) is included in the RREQ message along the path. After collection of all the RREQ messages the destination will select one route path which has less average transmission delay. The destination then construct a route reply message and reply along the path chosen.

Algorithm 2 Average delay in transmission (Minimization) Input: parameters in multi-hop CSN, destination SU D, source SU S

Output: path with the minimum expected transmission delay Source SU S creates RREQ messages m Source

SU S broadcasts $m$ through CCC channel

1: for all each $\mathrm{SU} n$ which receives $m$ do

2: if SU $\mathrm{n}$ is the destination SU D then

3: SU n obtains TTDp by Equation (8)

4: else

5: SU n obtains Ti,j by Equation (7)

6: SU $\mathrm{n}$ forwards $\mathrm{m}$ on over CCC channel

7: end if

8: end for

9: SU D waits for a predefined time upon receiving the first replica of message $\mathrm{m}$

10: SU D determines the best route path which has the minimum

TTDp by using Equation 10

11: SU D sends an acknowledgement back to $S$ along the chosen path.

\section{Pus Reclaim, Node mobility and failure}

Reclaim of licensed channels by primary users and SUs mobility leads to breaking of established routes. A node which moves out of range of its neighbors due to dynamic nature and PU reclaim will be detected, e.g. by link layer. In our work, affected nodes will start a limited route discovery starting from the affected node if it is second time interruption. We can reduce the disruption paths due to route breakage before the path is completely broken with the help of mobility models by estimating movement of the node. It should be noted that disrupted routes will expire by themselves and hence no action is required to remove them.

\section{Updation of Route}

With the primary user coming back there will be interruption in the data transmission which leads to rerouting and retransmission. Hence in this work, we consider that the next node which relays the information will remain same. For instance, two secondary users $i$ and $j$ are communicating over channel $\mathrm{k}$ will get interrupted on the arrival of primary user. If this interruption is happening second time for secondary user i during transmission, secondary user i will reroute using method 1 or method 2. If the interruption is first time for secondary user i during transmission, secondary user i will attempt for retransmission.

\section{RESULTS AND DISCUSSIONS}

A Cognitive radio based IoT environment is created and simulated using MATLAB software for the evaluation of 
routing performance by using our proposed routing algorithm. The comparison results are between static deployment, static deployment with local and global spectrum information [46] and proposed dynamic deployment with local and global spectrum information. The network topology is adopted from our previous work [51] where mobile relays were either pedestrian based relays, vehicular based relays or a combination of both. The simulations are run by 100 times by varying the number of nodes in the network and the confidence level is $95 \%$ which can be observed in [51 fig 2-fig 4]. Considering the dynamic nature of these mobile relays, we focused on capturing the effect of these mobile relays on the route sustenance and connection sustenance of an established connection. During setup phase by applying our proposed metrics (delivery success probability and transmission delay metric) the route is established between source and destination. Once the route is established it so happens that licensed user can come back or as the nodes are mobile the intended cognitive user relay may move out of coverage area. In [51] authors are going to get a reliable and feasible Cognitive User relays. In [51 fig 2-fig 6] by varying SNR's, considering path loss exponent and by varying the distance of mobile relay Cognitive users from the Base Station the above mentioned parameters success rate is presented. The results in [51 fig2-fig6] show that, by including the mobile relays beyond the coverage of network the route sustenance time and connection sustenance time improves substantially and also helps in extending the service area, network lifetime and performance of the network. With the help of this tested network [51], and by the inclusion of proposed routing metrics bit error rates are calculated

It is clearly evident from the following results that with the inclusion of enhanced energy detector [47] at each and every node has decreased the Bit error rate (BER) and increased signal to noise ratio (SNR) and throughput. As the route between source and destination is in a multi hop fashion, the BER between two neighboring nodes at the end of a link is denoted as $\mathrm{BER}_{\text {link, }}$, which depends on SNR ratio at the receiving node. It is observed from the considered network communication scenario with random topology a multi-hop route from a source to destination has $n_{h}$ links of lengths $r^{(i)}$ link $, i=1, \ldots, n_{h}$. Denoting by $\mathrm{BER}^{(\mathrm{i})}$ link the BER calculated at the end of the i-th link (the SNR at the receiving node of this link depends on the link length) assuming that (i) at each intermediate node there is regeneration; and that (ii) In successive links uncorrelated errors accumulate, it is possible to show that the BER at the end of the $n_{h}$-th link of the multi-hop route, denoted by BER ${ }^{\left(n_{h}\right)}$, can be expressed as [53] $\mathrm{BER}_{\text {link }}$

$$
\operatorname{BER}\left(n_{h}\right)=1-\prod_{i=1}^{n_{h}}\left[1-B E R_{\text {link }}(i)\right]
$$

We have considered the mobility model of nodes as GaussMarkov Model with temporal dependency [52]. In the simulation region we have considered 40 secondary users and 10 primary users. The average spectrum usage period of primary users varies from $0.5 \mathrm{~ms}$ to $25 \mathrm{~ms}$, we assumed bandwidth of each channel is $\mathrm{B}=0.5 \mathrm{MHz}$ and the data packet size is $2 \mathrm{~KB}$. We also assumed that, if it is not stated the detection probability is $90 \%$ and for better Quality of service the minimum data requirement is $0.3 \mathrm{bps} / \mathrm{Hz}$.

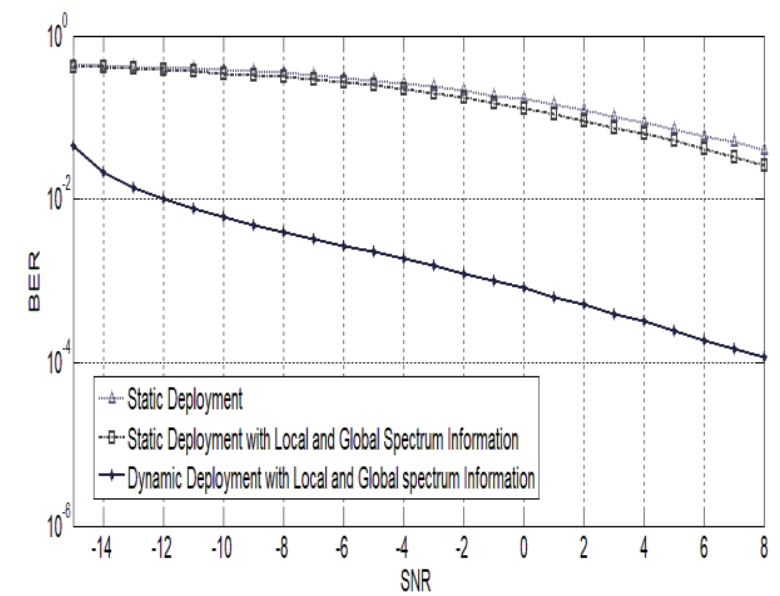

Fig. 1. BER vs. SNR of varying deployments with Spectrum information

From Figure 1 it is clearly observed that the BER of dynamic deployment with local and global spectrum information has decreased when compared with static deployment and static deployment with local and global spectrum information. This is due to usage of enhanced energy detector in the proposed algorithm at each and every node. Hence false alarms and missed detections are decreased and has impact on BERs. It is also observed that at low Signal to Noise Ratios (SNR) also our proposed algorithm is performing well.

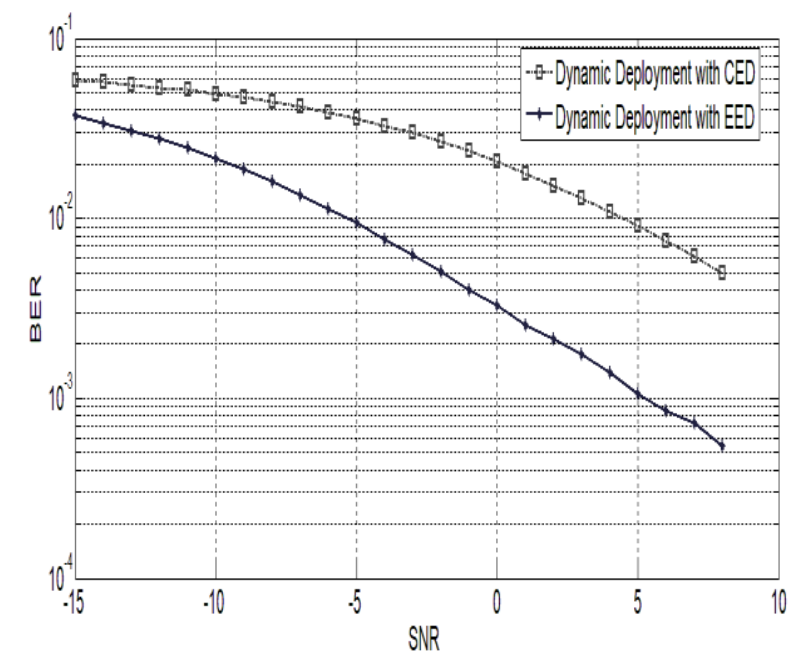

Fig. 2. BER vs. SNR with CED and EED at each and every node

In Figure 2 we have made comparison between dynamic deployment with conventional energy detector (CED) and dynamic deployment with enhanced energy detector (EED) [47]. In [47] probability of false alarms and enhancement of probability of detection is clearly discussed with CED and EED on cognitive femto cell networks. As probability of false alarms are more in CED the detection rate is less. If there is issue in the spectrum sensing itself then obviously disruption paths (PUs reclaim) will increase a lot and there is impact on BER which is evident from Figure 2. We have tested our routing algorithm on CED and EED on a dynamic deployment for comparison. The plot is analyzed between BER and SNR. It is observed that how 
enhancement in spectrum sensing algorithm plays a role in decreasing the BERs. In our proposed work, with the proposed routing metrics we simulated dynamic CR based IoT network with CED and EED to observe BERs.

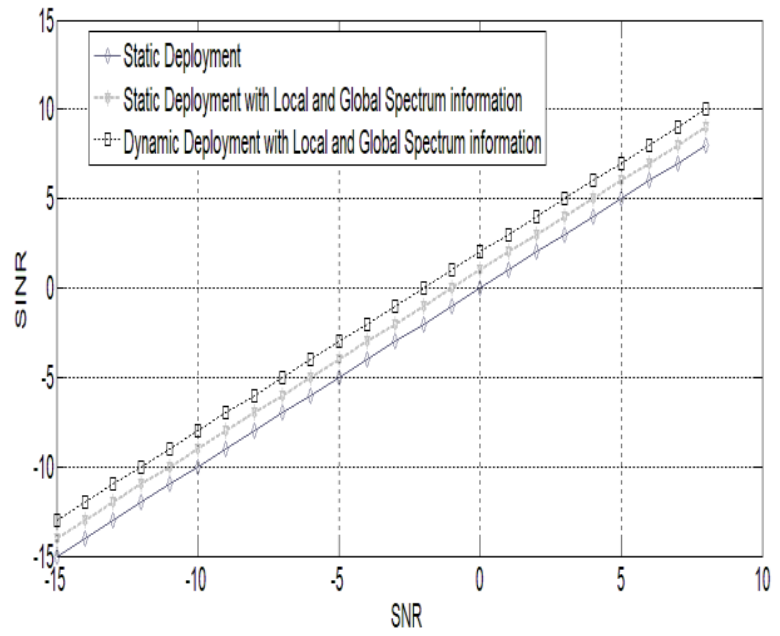

Fig. 3. SINR vs. SNR of varying deployments with local and global information

The Figure 3 is plotted for SINR and SNR between static deployment, static deployment with local and global spectrum information and dynamic deployment with local and global spectrum information. SINR is defined as the power of certain signal of interest divided by the sum of interference power from all other interfering signals and the power of some background noise. This is considered because the interference can happen with primary user coming back and due to false alarms and missed detections of spectrum sensing methods. As we have used EED the interferences caused due to false alarms and missed detections will be decreased and hence it is observed that dynamic deployment with local and global spectrum information has high SINRs when compared to existing and conventional methods.

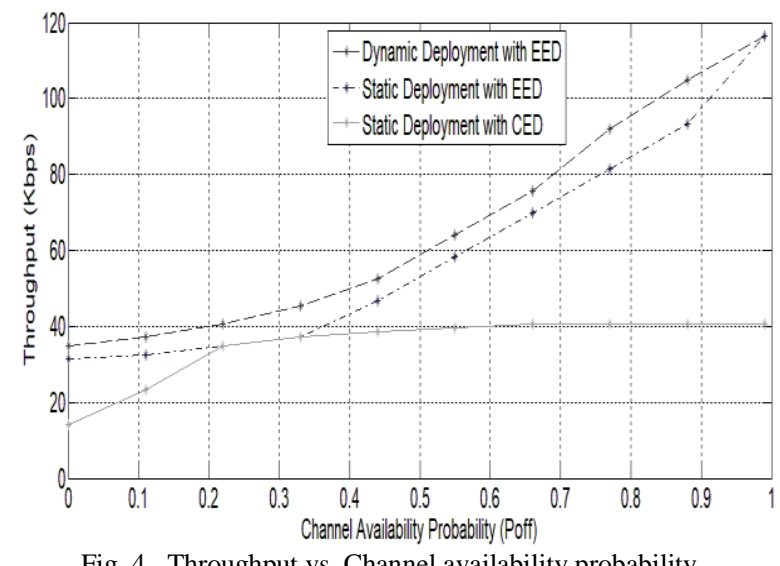

Fig. 4. Throughput vs. Channel availability probability of varying deployments

The Figure 4 is plotted between channel availability probability and throughput. It is observed from the plot that the throughput of dynamic deployment is more when compared with static deployment with EED and CED, with local and global spectrum information.

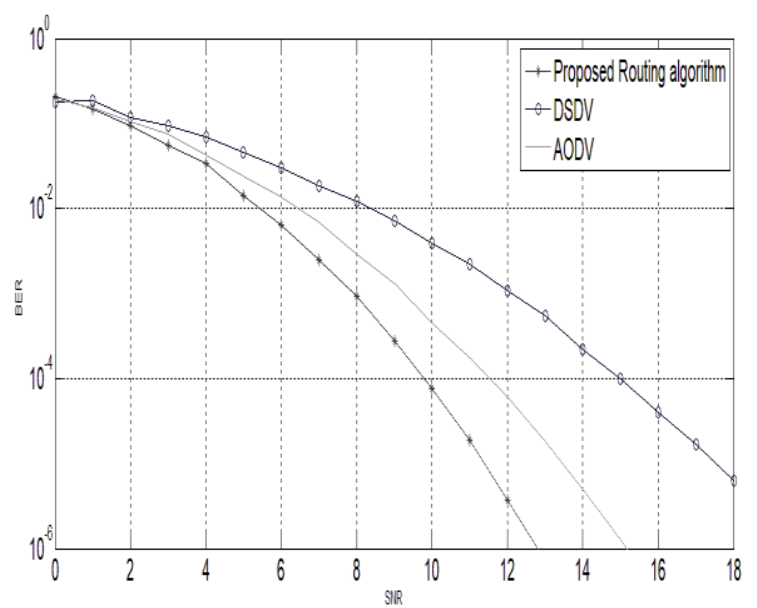

Fig. 5. Comparison of proposed routing protocol with existing ones

In the above figure we have made comparison between AODV, DSDV and our proposed routing algorithm with the routing metrics mentioned in section VII. AODV builds a route between nodes only if source nodes request the route and hence it is on-demand algorithm and it doesn't create any extra traffic along the communication link. Where as in Destination sequenced distance vector (DSDV), it is a table driven routing scheme and hence it requires frequent updates of its routing tables, which will use bandwidth and small amount of bandwidth even when the network is idle. In the proposed algorithm, the source secondary user will broadcast the route request message (RREQ) through common control channel in AODV way to destination secondary user. The success probability of each secondary user can be obtained by (6) is added along the path in RREQ message. Destination will select one route from number of RREQ messages by calculating the optimal path which has good success probability in delivering the data packets. Along the path which is been chosen with the help of route reply message it will reply back. During broadcasting information about spectrum regarding transmission delay by using (7) is included in the RREQ message along the path which is not the case in AODV and DSDV. After collection of all the RREQ messages the destination will select one route path which has less average transmission delay. The destination then construct a route reply message and reply along the path chosen. With the help of Equation 11 we have calculated the BERs of the routing protocols, and it is observed that the proposed algorithm outperforms the AODV and DSDV.

\section{CONCLUSION}

Due to the dynamicity in the availability of spectrum channels, routing will become difficult but an important issue to consider in Cognitive radio enabled IoT. In this work, by considering availability and quality of spectrum in the perspective of instant and global information about spectrum, two routing metrics are proposed in Cognitive radio enabled IoT. In the first metric by considering only one retransmission 
to decrease rerouting delivery success probability is defined. In the second metric average transmission delay over all channels is presented. The proposed routing algorithm is then designed by considering the above two metrics, then with the help of $\mathrm{ON}$ demand routing style optimal route is calculated. In this work we have considered dynamic nodes, and EED is applied at each and every node to minimize bit error rates and to maintain good SINR.

\section{REFERENCES}

[1] A. Al-Fuqaha, M. Guizani, M. Mohammadi, M. Aledhari and M. Ayyash, "Internet of Things: A Survey on Enabling Technologies, Protocols, and Applications," in IEEE Communications Surveys \& Tutorials, vol. 17, no. 4, pp. 2347-2376, Fourthquarter 2015. DOI 10.1109/COMST.2015.2444095

[2] P. Rawat, K. Singh, and J. Bonnin, "Cognitive radio for M2M and internet of things: A survey," Computer Communications, Vol. 94, pp. 1-29, 2016

[3] V. Petrov, S. Edelev, M. Komar and Y. Koucheryavy, "Towards the era of wireless keys: How the IoT can change authentication paradigm," 2014 IEEE World Forum on Internet of Things (WF-IoT), Seoul, 2014, pp. 5156. DOI: 10.1109/WF-IoT.2014.6803116

[4] H. A. Bany Salameh and M. Krunz, "Channel access protocols for multihop opportunistic networks: challenges and recent developments," in IEEE Network, vol. 23, no. 4, pp. 14-19, July-August 2009. DOI: 10.1109/MNET.2009.5191141

[5] J. Mitola and G. Q. Maguire, "Cognitive radio: making software radios more personal," in IEEE Personal Communications, vol. 6, no. 4, pp. 13 18, Aug. 1999.DOI: 10.1109/98.788210

[6] S. Haykin, "Cognitive radio: brain-empowered wireless communications," in IEEE Journal on Selected Areas in Communications, vol. 23, no. 2, pp 201-220, Feb. 2005. DOI: 10.1109/JSAC.2004.839380

[7] B. Wang and K. J. R. Liu, "Advances in cognitive radio networks: A survey," in IEEE Journal of Selected Topics in Signal Processing, vol. 5 , no. 1, pp. 5-23, Feb. 2011. DOI: 10.1109/JSTSP.2010.2093210

[8] M. Zareei, E. Mahmoud Mohamed, M. H. Anisi, C. Vargas Rosales, K. Tsukamoto and M. Khurram Khan, "On-Demand Hybrid Routing for Cognitive Radio Ad-Hoc Network," in IEEE Access, vol. 4, pp. 8294 8302, 2016. DOI: 10.1109/ACCESS.2016.2626721

[9] O. B. Akan, O. B. Karli and O. Ergul, "Cognitive radio sensor networks," in IEEE Network, vol. 23, no. 4, pp. 34-40, July-August 2009. DOI 10.1109/MNET.2009.5191144

[10] M. Yan, S. Ji, M. Han, Y. Li and Z. Cai, "Data aggregation scheduling in wireless networks with Cognitive Radio capability," 2014 Eleventh Annual IEEE International Conference on Sensing, Communication, and Networking (SECON), Singapore, 2014, pp. 513-521.DOI: 10.1109/SAHCN.2014.6990390

[11] Z. Yang, Z. Shi, and C. Jin, "SACRB-MAC: A high-capacity MAC protocol for cognitive radio sensor networks in smart grid," Sensors, vol. 16, no. 4, p. 464, 2016

[12] S. Zubair, S. K. S. Yusoff, and N. Fisal, “Mobility-enhanced reliable geographical forwarding in cognitive radio sensor networks," Sensors, vol. 16 , no. 2 , p. 172,2016

[13] G. P. Joshi and S.W. Kim, “A survey on node clustering in cognitive radio wireless sensor networks," Sensors, vol. 16, no. 9, p. 1465, 2016

[14] A. R. Syed, K. A. Yau, J. Qadir, H. Mohamad, N. Ramli and S. L. Keoh, "Route Selection for Multi-Hop Cognitive Radio Networks Using Reinforcement Learning: An Experimental Study," in IEEE Access, vol. 4, pp. 6304-6324, 2016. DOI: 10.1109/ACCESS.2016.2613122

[15] G. P. Joshi, S. Y. Nam, and S. W. Kim, “'Cognitive radio wireless sensor networks: Applications, challenges and research trends," Sensors, vol. 13 no. 9, pp. 11196-11228, 2013

[16] L. Zhang, Z. Cai, P. Li, and X. Wang, “'Exploiting spectrum availability and quality in routing for multi-hop cognitive radio networks," in Proc 11th Int. Conf. Wireless Algorithms, Syst., Appl. (WASA), Aug. 2016, pp 283-294.

[17] K. Wei, X. Liang and K. Xu, "A Survey of Social-Aware Routing Protocols in Delay Tolerant Networks: Applications, Taxonomy and Design-Related Issues," in IEEE Communications Surveys \& Tutorials, vol. 16, no. 1, pp. 556-578, First Quarter 2014. DOI: 10.1109/SURV.2013.042313.00103
[18] L. Zhang, X. Wang, J. Lu, M. Ren, Z. Duan, and Z. Cai, “A novel contact prediction-based routing scheme for DTNs," Trans. Emerg. Telecommun. Technol., vol. 28, no. 1, pp. e2889-1-e2889-12, 2017.

[19] L. Zhang, Z. Cai, J. Lu, and X. Wang, “'Mobility-aware routing in delay tolerant networks," Pers. Ubiquitous Comput., vol. 19, no. 7, pp. 1111$1123,2015$.

[20] R. Zhao, X. Wang, Y. Lin, Y. Yang, T. Hui, and L. Zhang, “ A controllable multi-replica routing approach for opportunistic networks," IEEJ Trans. Elect. Electron. Eng., 2017 doi: 10.1002/tee.22437.

[21] S. Sengupta and K. P. Subbalakshmi, "Open research issues in multi-hop cognitive radio networks," in IEEE Communications Magazine, vol. 51, no. 4, pp. 168-176, April 2013. DOI: 10.1109/MCOM.2013.6495776

[22] M. Cesana, F. Cuomo, and E. Ekici, ' 'Routing in cognitive radio networks Challenges and solutions," Ad Hoc Netw., vol. 9, no. 3, pp. 228-248, 2011.

[23] K. R. Chowdhury and M. D. Felice, "Search: A routing protocol for mobile cognitive radio ad-hoc networks," Comput. Commun., vol. 32, no. 18, pp. 1983-1997, 2009.

[24] A. A. Khan, M. H. Rehmani and A. Rachedi, "When Cognitive Radio meets the Internet of Things?," 2016 International Wireless Communications and Mobile Computing Conference (IWCMC), Paphos, 2016, pp. 469-474. DOI: 10.1109/IWCMC.2016.7577103

[25] M. Zhang et al., "Cognitive Internet of Things: Concepts and Application Example," IJCSI Int'l. J. Computer Science Issues, vol. 9, no. 6, Nov. 2012, pp. 151-58.

[26] Y. H. Lin, Q. Wang, J. S. Wang, L. Shao and J. Tang, "Wireless IoT Platform Based on SDR Technology," 2013 IEEE International Conference on Green Computing and Communications and IEEE Internet of Things and IEEE Cyber, Physical and Social Computing, Beijing, 2013, pp. 2245-2246 DOI: 10.1109/GreenCom-iThings-CPSCom.2013.426

[27] R. F. Shigueta, M. Fonseca, A. C. Viana, A. Ziviani and A. Munaretto, "A strategy for opportunistic cognitive channel allocation in wireless Internet of Things," 2014 IFIP Wireless Days (WD), Rio de Janeiro, 2014 pp. 1-3. DOI: 10.1109/WD.2014.7020850

[28] E. Z. Tragos and V. Angelakis, "Cognitive Radio Inspired M2M Communications," 16th Int'l. Symp. Wireless Personal Multimedia Commun., 2013.

[29] Q. Wu et al., "Cognitive Internet of Things: A New Paradigm Beyond Connection," in IEEE Internet of Things Journal, vol. 1, no. 2, pp. 129143, April 2014. DOI: 10.1109/JIOT.2014.2311513

[30] D. Kelaidonis et al., Eds., "Virtualization and Cognitive Management of Real World Objects in the Internet of Things," Proc. Int'l. Conf. Internet of Things, Besanbon, France, Nov. 20-23 2012.

[31] A. Somov, C. Dupont, and R. Giaffreda, "Supporting Smart-City Mobility with Cognitive Internet of Things," Future Network and Mobile Summit, 2013.

[32] V. Miz and V. Hahanov, "Smart Traffic Light in Terms of the Cognitive Road Traffic Management System (CTMS) Based on the Internet of Things," East-West Design and Test Symp., 2014.

[33] O. S. Badarneh and H. B. Salameh, "Opportunistic Routing in Cognitive Radio Networks: Exploiting Spectrum Availability and Rich Channel Diversity," 2011 IEEE Global Telecommunications Conference GLOBECOM 2011, Kathmandu, 2011, pp. 1-5. DOI: 10.1109/GLOCOM.2011.6134241

[34] X. Jin, R. Zhang, J. Sun and Y. Zhang, "TIGHT: A Geographic Routing Protocol for Cognitive Radio Mobile Ad Hoc Networks," in IEEE Transactions on Wireless Communications, vol. 13, no. 8, pp. 4670-4681, Aug. 2014. doi: 10.1109/TWC.2014.2320950

[35] Y. Liu, L. X. Cai and X. S. Shen, "Spectrum-Aware Opportunistic Routing in Multi-Hop Cognitive Radio Networks," in IEEE Journal on Selected Areas in Communications, vol. 30, no. 10, pp. 1958-1968, November 2012. doi: 10.1109/JSAC.2012.121111

[36] A. M. E. Ejmaa, S. Subramaniam, Z. A. Zukarnain and Z. M. Hanapi, "Neighbor-Based Dynamic Connectivity Factor Routing Protocol for Mobile Ad Hoc Network," in IEEE Access, vol. 4, pp. 8053-8064, 2016. doi: 10.1109/ACCESS.2016.2623238

[37] Z. Cai, Y. Duan, and A. G. Bourgeois, “Delay efficient opportunistic routing in asynchronous multi-channel cognitive radio networks," $J$. Combinat.Optim., vol. 29, no. 4, pp. 815-835, 2015.

[38] X. Wang, Y. Lin, Y. Zhao, L. Zhang, J. Liang, and Z. Cai, “A novel approach for inhibiting misinformation propagation in human mobile opportunistic networks," Peer-Peer Netw. Appl., vol. 10, no. 2, pp. 377394, 2017.

[39] S. Cheng, Z. Cai, J. Li and H. Gao, "Extracting Kernel Dataset from Big Sensory Data in Wireless Sensor Networks," in IEEE Transactions on 
Knowledge and Data Engineering, vol. 29, no. 4, pp. 813-827, 1 April 2017. doi: 10.1109/TKDE.2016.2645212

[40] X. Guan, A. Li, Z. Cai, and T. Ohtsuki, “Coalition graph game for robust routing in cooperative cognitive radio networks," Mobile Netw. Appl., vol. 20, no. 2, pp. 147-156, 2015

[41] Z. Cai, S. Ji, J. He and A. G. Bourgeois, "Optimal Distributed Data Collection for Asynchronous Cognitive Radio Networks," 2012 IEEE 32nd International Conference on Distributed Computing Systems, Macau, 2012, pp. 245-254. DOI: 10.1109/ICDCS.2012.29

[42] S. Ji, Z. Cai, J. S. He and R. Beyah, "Primary social behavior aware routing and scheduling for Cognitive Radio Networks," 2015 12th Annual IEEE International Conference on Sensing, Communication, and Networking (SECON), Seattle, WA, 2015, pp. 417-425. DOI: 10.1109/SAHCN.2015.7338342

[43] C. Perkins, E. Belding-Royer, and S. Das, Ad Hoc On-Demand Distance Vector (AODV) routing, document RFC 3561, 2003, pp. 1-5.

[44] W. Feng, J. Cao, C. Zhang and C. Liu, "Joint Optimization of Spectrum Handoff Scheduling and Routing in Multi-hop Multi-radio Cognitive Networks," 2009 29th IEEE International Conference on Distributed Computing Systems, Montreal, QC, 2009, pp. 85-92. DOI: 10.1109/ICDCS.2009.64

[45] S. Ji, M. Yan, R. Beyah and Z. Cai, "Semi-Structure Routing and Analytical Frameworks for Cognitive Radio Networks," in IEEE Transactions on Mobile Computing, vol. 15, no. 4, pp. 996-1008, 1 April 2016. DOI: $10.1109 /$ TMC.2015.2442250

[46] L. Zhang, Z. Cai, P. Li, L. Wang and X. Wang, "Spectrum-Availability Based Routing for Cognitive Sensor Networks," in IEEE Access, vol. 5, pp. 4448-4457, 2017. DOI: 10.1109/ACCESS.2017.2681743

[47] M. K. Kaushik, T. S. Sai and Y. Yoganandam, "Enhanced energy detection and moving average thresholding for cognitive femtocell networks," 2013 IEEE Asia Pacific Conference on Postgraduate Research in Microelectronics and Electronics (PrimeAsia), Visakhapatnam, 2013, pp. 250-253. DOI: 10.1109/PrimeAsia.2013.6731215.

[48] H. A. Bany Salameh, M. Krunz and O. Younis, "Cooperative Adaptive Spectrum Sharing in Cognitive Radio Networks," in IEEE/ACM Transactions on Networking, vol. 18, no. 4, pp. 1181-1194, Aug. 2010. DOI: $10.1109 /$ TNET.2009.2039490

[49] S. Bayhan and F. Alagöz, "A Markovian approach for best-fit channel selection in cognitive radio networks," Ad Hoc Netw., vol. 12, pp. 165177, Jan. 2014

[50] S. Stotas and A. Nallanathan, "On the Outage Capacity of Sensing Enhanced Spectrum Sharing Cognitive Radio Systems in Fading Channels," in IEEE Transactions on Communications, vol. 59, no. 10, pp. 2871-2882, October 2011. DOI: 10.1109/TCOMM.2011.063011.100787

[51] M. K. Kaushik and Y. Yoganandam, "Studies on route sustenance and outage probability in cognitive mobile secondary user networks," 2016 Sixth International Symposium on Embedded Computing and System Design (ISED), Patna, 2016, pp. 245-248. DOI: 10.1109/ISED.2016.7977090

[52] F. Bai, Narayanan Sadagopan and A. Helmy, "IMPORTANT: a framework to systematically analyze the Impact of Mobility on Performance of Routing Protocols for Adhoc Networks," IEEE INFOCOM 2003. Twentysecond Annual Joint Conference of the IEEE Computer and Communications Societies (IEEE Cat. No.03CH37428), San Francisco, CA, 2003, pp. 825-835 vol.2. DOI: 10.1109/INFCOM.2003.1208920

[53] N. Wisitpongphan, G. Ferrari, S. Panichpapiboon, J. S. Parikh and O. K. Tonguz, "QoS provisioning using BER-based routing in ad hoc wireless networks," 2005 IEEE 61st Vehicular Technology Conference, Stockholm, 2005, pp. 2483-2487 Vol. 4 DOI: 10.1109/VETECS.2005.1543782

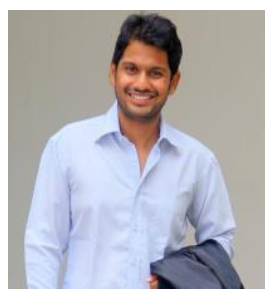

M.K. Kaushik received the B.E. degree in Electronics and communications engineering from NCET, VTU, Bangalore, in 2008 and the M.Tech. degree in Communications and Signal Processing from GPREC, SKU, Kurnool, in 2010. He is currently pursuing the Ph.D. degree in Department of Electrical Engineering at BITS Pilani Hyderabad Campus, Hyderabad. From 2010 to 2012, he was a Senior Technologist with the Center for Integrated

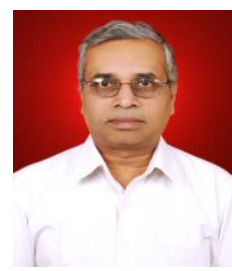

Yoganandam Yeleswarapu was in academics for 24 years and in aeronautics, telecommunications product industries for about 13 years and associated for 2 years with the Signal Processing research group at Electrical and Computer Engineering department, Concordia University, Montreal, Canada. He completed his B.Tech in Electronics \& Communications Engineering, JNT University, Hyderabad, India (1977). He obtained his M.Tech in Microwave \& Radar Engineering, IIT, Kharagpur, India (1979) He received his Ph.D degree in Electronics \& Communications Engineering, Osmania University, India (1987).

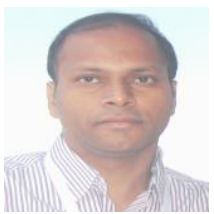

Subhend. K. Sahoo completed his B.E. in Electronics and telecommunication engineering from Utakal University, Orissa, India in the year 1994 with honours securing fifth position in the university. He obtained his M.E. in Electronic Systems and Communication from R.E.C.(NIT) Rourkela in 1998. He received the Ph. D. degree in electrical engineering from Birla Institute of Technology and Science, Pilani, in 2006. He was working as a assistant professor then associate professor in Electrical and Electronics Engineering department from 2009 till 2018 in Birla Institute of Technology and Science, Pilani. Presently he is working as associate professor and in SPM International Institute of Information Technology, Naya Raipur, India. His areas of research are high performance arithmetic circuits and VLSI circuits. 